\title{
Bone Marrow-Derived Stem Cells: a Mixed Blessing in the Multifaceted World of Diabetic Complications
}

\author{
Giuseppe Mangialardi ${ }^{1} \cdot$ Paolo Madeddu $^{1}$ \\ Published online: 30 March 2016 \\ (C) The Author(s) 2016. This article is published with open access at Springerlink.com
}

\begin{abstract}
Diabetes is one of the main economic burdens in health care, which threatens to worsen dramatically if prevalence forecasts are correct. What makes diabetes harmful is the multi-organ distribution of its microvascular and macrovascular complications. Regenerative medicine with cellular therapy could be the dam against life-threatening or life-altering complications. Bone marrow-derived stem cells are putative candidates to achieve this goal. Unfortunately, the bone marrow itself is affected by diabetes, as it can develop a microangiopathy and neuropathy similar to other body tissues. Neuropathy leads to impaired stem cell mobilization from marrow, the so-called mobilopathy. Here, we review the role of bone marrow-derived stem cells in diabetes: how they are affected by compromised bone marrow integrity, how they contribute to other diabetic complications, and how they can be used as a treatment for these. Eventually, we suggest new tactics to optimize stem cell therapy.
\end{abstract}

Keywords Diabetes - Diabetic complications - Bone marrow $\cdot$ Stem cells $\cdot$ Microangiopathy $\cdot$ Cell regenerative therapy

This article is part of the Topical Collection on Immunology and Transplantation

Paolo Madeddu

paolo.madeddu@bristol.ac.uk

Giuseppe Mangialardi

giuseppe.mangialardi@bristol.ac.uk

1 Bristol Heart Institute, University of Bristol, Level 7, Bristol Royal Infirmary, Upper Maudlin Street, Bristol BS28HW, UK

\section{Introduction}

Diabetes mellitus (DM) is a family of metabolic disorders characterized by high blood glucose levels. Worldwide, there are about 387 million people affected by DM, with a prevalence in North America of $11.4 \%$ (39 million) and in Europe of $7.9 \%$ (52 million) (http://www.idf.org/sites/default/files/ DA-regional-factsheets-2014_FINAL.pdf). Estimation for 2035 foresees 592 million patients with diabetes on the planet. It is not surprising that DM is one of the heaviest economic health burdens, with an annual global cost of 548 million USD raising to 627 million in 2035 (http://www.idf. org/sites/default/files/DA-regional-factsheets-2014_FINAL. pdf).

There are several types of DM. Type 1 diabetes (T1DM) is an autoimmune disease characterized by $\beta$ cell destruction in the pancreatic islets resulting in the complete loss of insulin secretion. Type 2 diabetes (T2DM), the prevalent form of DM, is a multifactorial disease characterized by insulin resistance. Gestational diabetes (GDM) occurs in pregnant women without previous history of DM. Chronic hyperglycemia causes systemic complications, with cardiovascular complications being particularly frequent and worrisome [1]. Diabetic macrovascular disease manifests as coronary artery disease (CAD), myocardial infarction (MI), peripheral artery disease (PAD), and stroke. Microvascular disease results from damage to the small vessels and can aggravate the outcome of patients with occlusive macrovascular disease. Compared to hospital costs of people without complications, hospital costs of those with microvascular complications are doubled, those of people with macrovascular complications are tripled, and those of people with both micro- and macrovascular complications are six times as great. Lifestyle interventions, such as changes in diet and 
exercise, and medications are essential in reducing the risk for development of complications. Thanks to improved management, the prevalence of diabetic complications is constantly decreasing, but these improved clinical outcomes came with a cost of $£ 708$ million per year in the UK, corresponding to $7 \%$ of the UK health-care prescribing budget [2]. Nevertheless, once cardiovascular complications occur, these are more difficult to treat in patients with DM as compared with patients without DM. This difference has been explained with the fact that healing and regenerative mechanisms are remarkably altered in patients with DM.

The current care of cardiovascular complications comprises pharmacotherapy and revascularization. However, medical treatment can be ineffective as in the case of refractory angina, which is prevalent in patients with DM. Additionally, in many patients with DM, revascularization cannot be applied due to multiple and distal occlusions, or it fails because of restenosis. But the most important limitation of medical and interventional treatments is that, even when successful, they do not replace cells irreversibly damaged by ischemia. Therefore, intense research is focused on regenerative medicine as a novel option for the treatment of cardiovascular complications. This approach consists of augmenting endogenous mechanisms of repair by supplements of regenerative cells, for instance through transplantation of stem cells/ progenitor cells into the infarcted myocardium. Alternatively, cytokines and growth factors could be delivered to boost the liberation of progenitor cells from the bone marrow (BM) or the expansion of resident progenitor cells in the heart and other ischemic tissues. To date, a few thousand individuals worldwide have received stem cells - mainly BM cells-in cardiovascular clinical trials [3].

In this review, we provide a concise report of the latest findings about cell therapy for treatment of diabetic complications, with particular focus on approaches based on BMderived cells. We also discuss the pros and cons of BM cell therapy, especially in light of new discoveries that common complications of DM, namely microangiopathy and neuropathy, affect also the BM and may jeopardize stem cell mobilization following an ischemic event or stimulation by cytokines. Finally, we propose some possible solutions to improve current cell therapy approaches.

\section{Bone Marrow-Derived Stem Cells and Their Niches}

$\mathrm{BM}$ is the only reservoir of human pluripotent stem cells (HPSCs) in the adult human body, providing a specific microenvironment to support stem cell maintenance and/or expansion. Here, we provide a brief overview of the different cell populations of interest and their organization in the BM niche.

\section{Hematopoietic Stem Cells}

Hematopoietic stem cells (HSCs) constitute a very heterogeneous population, which can give rise to any blood cell. They are defined as proper stem cells because of their self-renewal capacity and multipotency [4]. BM transplantation assays in animal models helped to establish a possible hierarchical structure. Accordingly, based on repopulating capacity upon transplantation into irradiated animals, two different populations have been identified and defined as short-term HSCs (ST-HSC) and long-term HSCs (LT-HSCs) [5]. In humans, the surface marker $\mathrm{CD} 34$, a member of a family of singlepass transmembrane glycoproteins, is considered the main defining marker for HSCs and the marker of choice to select populations for clinical cell therapy trials. Depleting CD34+ cells for Lin antigen enables their enrichment into a more primitive population with hematopoietic function. Further enrichment could be achieved by selecting the latter population for Thy1, a primitive stem cell marker, and depleting for CD38 and CD45RA markers (multipotent progenitors), with additional positivity for CD49f defining proper LT-HSCs [6]. In the mouse, LT-HSCs are identified as CD34-/c-Kit+/Sca1+/Lineage- (CD34-/KSL) cells. However, CD34-/KSL cells represent a heterogeneous population containing subfractions with diverse regenerative capacity. Further characterization by use of the signaling lymphocyte activation molecule (SLAM) markers CD48 and CD150 allows the identification of a fraction of CD34-/KSL cells endowed with high self-renewal potential and repopulating capacity [4]. The seminal concept of BM HSCs directly participating in de novo post-natal vasculogenesis, proposed some years ago by Asahara et al. [7], has been revised in light of the novel indication that paracrine mechanisms, rather than the conversion of one cell type to another, control the interrelation between hematopoietic and vascular cells [8].

\section{Mesenchymal Stem Cells}

Mesenchymal stem cells (MSCs) are a heterogeneous multipotent stromal cell population well represented in BM as well as in several other adult tissues. They are defined as plastic-adherent, fibroblast-like cells endowed with high proliferative activity and capacity to differentiate into a variety of cell types, including osteoblasts (bone cells), chondrocytes (cartilage cells), myocytes (muscle cells), and adipocytes (fat cells) [9]. They typically express the mesenchymal markers CD44, CD90, CD49, CD54, CD105, and CD73, but are negative for both hematopoietic markers, such as CD45, CD14, and $\mathrm{CD} 11 \mathrm{~b}$, and endothelial markers, such as CD144, von Willebrand factor (vWf), and VCAM-1 [9]. MSCs have been shown to possess special plasticity. In fact, under appropriate experimental conditions, they can also differentiate into nonmesenchymal cells - for instance, neural cells and endothelial 
cells [10]. In addition, MSCs exert pro-angiogenic and immunomodulatory activities, through the secretion of different cytokines/growth factors [11], altogether making MSCs a popular choice for regenerative medicine applications.

\section{Endothelial Progenitor Cells}

Endothelial progenitor cells (EPCs) are angiogenic cells characterized for the first time by Ishikawa and Asahara in 1997 [12]. Since their discovery, many definitions have been proposed, creating a maelstrom of overlapping nomenclatures. Scientific consensus agreed on defining EPCs as a subset of myeloid/monocyte cells which can acquire endothelial markers, such as CD31, KDR, or vWf, together with lectinbinding ability in in vitro culture conditions [13]. They have to be considered a different population from endothelial colonyforming cells (ECFCs), a non-marrow-derived population, and more resembling endothelial cells likely originated from vessel-associated progenitor cells [14]. EPCs exert their proangiogenic potential in a paracrine fashion rather than differentiating in vascular cytotype [15]. EPCs have been extensively studied in animal models and clinical trials thanks to their supportive role in angiogenesis.

\section{Localization of Stem Cells in the BM Niche}

The BM is found within the cavities of long bones. The trabecular bone structure endowed with spare adipocytes provides a supportive meshwork for niches. Two different types of niches have been identified so far. The osteoblastic niche localized near the endosteal part of the bone provides a shelter for most primitive HSCs. A mixture of different cells contributes to the maintenance of HSC homeostasis. In particular, osteoblasts and macrophages play a pivotal role in regulating the endosteal niche milieu. Osteoblasts regulate the niche through Wnt signaling, and their depletion leads to niche pauperization [16]. A particular subset of macrophages, expressing CD169, could regulate the abundance of osteoblasts and also regulate the retentionmobilization of HSCs [17]. The vascular niche is organized around sinusoidal vessels and harbors more committed HSCs. In this niche, endothelial cells and perivascular cells are the main regulators. Endothelial cells mediate maintenance of HSC homeostasis through production of Akt-related angiocrine factors, such as angiopoietin-1, delta-like 1, insulin-like growth factorbinding protein 2, and epidermal growth factor [18]. Perivascular cells positive for CD146, platelet growth factor receptor $\alpha$, and nestin could be recognized around the sinusoids. They express two master regulators of HSC homeostasis: the chemokine (C-X-C motif) ligand 12 (CXCL-12) and stem cell factor (SCF) [19]. It has also been observed that perivascular cells exert their effects through cell-to-cell contacts via the Notch signaling pathway [19]. This spatial organization creates a gradient, in which HSCs could progress from the most primitive self-renewal state to lineage commitment, ready for egression into the bloodstream. Additionally, reactive oxygen species (ROS) distribute according to the BM perfusion gradient, with higher levels in the vascular niche and lower levels in the endosteal niche [20•]. This gradient contributes in maintaining the balance between stem cell self-renewal and differentiation across the niches $\left[20^{\bullet}\right]$.

\section{The Upside: Evidence for BM-Derived Stem Cells Improving Diabetic Complications}

\section{Diabetic Retinopathy}

Diabetic retinopathy (DR) is the major cause of vision impairment in working-age adults in western countries [21]. The main accepted mechanism in DR development is related to the loss of pericytes. They provide a nourishing, anti-inflammatory, and stabilizing microenvironment for human retinal endothelial cells (HREC). In the early stage, DM impairs pericyte self-renewal and endothelial cell survival, resulting in a hypoxic retinal microenvironment [22]. This triggers the translocation of hypoxia-inducible factor-1 alpha (HIF-1a), which in turn leads to the upregulated expression of vascular endothelial growth factor A (VEGF-A). Persistent VEGF-A production stimulates retinal angiogenesis, fibroblast scar formation, and vascular leakage, resulting in proliferative diabetic retinopathy (PDR) [22].

Cell therapy has been proposed as a novel approach to treat diabetic PDR. In this context, MSCs emerged as one of the most promising candidates for several reasons. A large body of evidence points out an equivalence between MSCs and pericytes [23], making MSCs the ideal replacement for the latter. Moreover, BM-derived MSCs have the ability to partially differentiate into photoreceptors and retinal pigmented epithelium in vitro and in vivo [24]. This ability is important considered that neurodegenerative events, including glial cell reactivity, microglial activation, and neuronal apoptosis, take place at the early stage of DR [25]. First phase I/II clinical trials using adult MSCs have been carried out (https://www.clinicaltrials.gov, NCT01068561 and NCT01560715) in retinitis pigmentosa. In the RETICELL clinical study, Siqueira et al. demonstrated an improvement in patient quality of life, unfortunately only for a short time, as any beneficial effect had disappeared after 12 months [26]. Currently, only two clinical trials with intravitreal injection of adult MSCs in patients suffering from ischemic retinopathy and 
macular degeneration are ongoing (https://www. clinicaltrials.gov, NCT01518842 and NCT01518127).

Besides MSCs, CD34+ HSCs represent a valid alternative. In different animal models, intravitreally injected CD34+ cells showed, similarly to MSCs, the ability to rescue retinal vasculature and a neuronal protective effect [27]. More importantly, intravitreal injection of BM-derived CD34+ cells is safe and well tolerated on the long term [27]. Park et al. detected CD34+ cells after 8 months from injections, with no histological sign of intraocular tumor or abnormal tissue growth [28]. Those premises paved the way to the first clinical trial (https:// www.clinicaltrials.gov, NCT01736059) in patients who are irreversibly blind from various retinal conditions, including DR. In vivo imaging showed changes suggestive of new cellular incorporation into the macula of the hereditary macular degeneration study [29]. Previously, Siqueira et al. treated patients with macular edema associated with macular ischemia, observing an improvement in patient visual performances [30].

\section{Diabetic Cardiomyopathy}

Diabetic cardiomyopathy (DCM) was firstly described in 1972 by Rubler et al. who observed congestive heart failure in patients with no coronary artery disease (CAD) [31]. The definition of DCM has evolved through the years and to date comprises the presence of diabetic microangiopathy, myopathy, and autonomic neuropathy. Briefly, DCM is characterized by an increased left ventricular (LV) mass and wall thickness frequently associated with cardiomyocyte hypertrophy [31]. Similar to other diabetic complications, DCM likely originates from altered metabolism related to chronic hyperglycemia. Insulin resistance leads to a switch from glucose oxidation metabolism to free fatty acid oxidation which is more oxygen demanding. Those events trigger cardiac ischemia inducing the shift to anaerobic metabolism, which in turn produces more lactate and acid metabolites resulting in lipid accumulation and altered cardiomyocyte contraction and apoptosis [32]. Increased oxidative stress and advanced glycation end product (AGE) formation contribute to fibrosis and cell death, while the occurrence of endothelial dysfunction manifests as altered permeability and increased leukostasis, leading to myocardial edema and inflammation [32].

Cell therapy is expected to benefit DCM through regenerative, pro-angiogenic, anti-fibrotic, and anti-inflammatory mechanisms. MSCs showed the in vitro and in vivo ability to differentiate into cardiomyocytes [33]. In the seminal work by Nagaya et al., MSC therapy was able to significantly increase capillary density and to decrease the collagen volume fraction in the myocardium, resulting in decreased left ventricular end-diastolic pressure [33]. The protective effect exerted by MSCs is seemingly attributed to remarkable paracrine activity. For instance, in vivo studies showed that intra-cardiac injection of MSCs in a rat model of post-ischemic heart failure led to a significant decrease in ventricular fibrosis associated with improved cardiac parameters. The effect was mainly mediated by the inhibition of MMP-9, a mediator of apoptosis in DCM, and the promotion of MMP-2 activity, which inhibits collagen deposition [34]. Additionally, MSCs protect cardiomyocytes from apoptosis by upregulating 14-3-3 and p-Ask1 proteins [35]. Moreover, MSCs are able to decrease immune cell infiltration [36]. Cytokines and growth factors could be used to potentiate stem cell mobilization and homing to the myocardium. Improved mobilization with granulocyte colony-stimulating factor (G-CSF) ameliorates the rat model of CDM decreasing perivascular interstitial fibrosis [37]. Our recent study in BM cells from infarcted patients demonstrated that SDF-1 directed migration enriches for cells with increased healing activity [38]. Additionally, BM-derived EPCs not only promote neovascularization but also exert anti-fibrotic action in diabetic hearts by paracrine inhibition of miR-155 [39].

To date, BM cell therapy has been tested in several clinical trials on patients with acute or chronic heart failure, producing mixed results [40]. However, to the best of our knowledge, no clinical trial has been so far conducted with BM cells for treatment of DCM.

\section{Diabetic Nephropathy}

Diabetic nephropathy is the leading cause of end-stage renal disease (ESRD) worldwide. Diabetic kidney disease is characterized by glomerular hypertrophy, basement membrane thickening, mesangial expansion, tubular atrophy, interstitial fibrosis, and arteriolar thickening. Those morphological changes are attributable to chronic hyperglycemia and enhanced oxidative stress. Podocyte dysfunction is key in these pathological process.

BM cell therapy has been tested in animal models of diabetic nephropathy with promising results. In 2006, Lee et al. reported that intracardial injection of MSCs in a T1DM mouse model improves metabolic control as well as glomerular morphology, mesangial thickening, and macrophage infiltration [41]. In a murine model of DM, Ezquer et al. obtained similar results, even if no restoration of normal glucose levels was observed. Interestingly, MSCs were detectable after 3 months of injection [42]. In a rat model of diabetic nephropathy, improved podocyte function was paralleled by decreased albuminuria [43]. Similar studies in a diabetic rat model provided mechanistic insight of autologous MSC transplant. The renoprotective mechanism of MSC therapy includes inhibition of oxidative stress, possibly through improved insulin sensitivity leading to better metabolic control [44]. Furthermore, rat bone marrow-derived MSCs decrease macrophage infiltration by downregulation of cytokines, like monocyte chemotactic protein 1 (MCP-1), interleukin $1 \beta$ (IL-1 $\beta$ ), interleukin 6 (IL-6), and tumor necrosis factor $\alpha(\mathrm{TNF}-\alpha)$ [45]. Additionally, 
VEGF and anti-apoptotic protein B cell lymphoma 2 (bcl2) are upregulated and transforming growth factor $\beta$ (TGF- $\beta$ ) and pro-apoptotic factor Bax are downregulated [46]. Inhibition in the TGF- $\beta$ signaling pathway is associated with decreased fibrosis, with this mechanism being likely mediated by secreted protein osteogenic protein-1 (OP-1) [47]. A study from Zhang et al. showed similar results in $\mathrm{a} d \mathrm{~d} / \mathrm{db}$ mouse model injected with EPCs [48]. Finally, recruitment of healing BM cells could occur in the beneficial effects of angiotensin receptor antagonists in preventing diabetic glomerulosclerosis [49].

\section{Diabetic Neuropathy}

Diabetic neuropathy (DNP) is a common and disabling manifestation of DM and often overlaps with vascular complications. Sensory neuropathy is a typical form of peripheral neuropathy characterized by an altered perception of noxious stimuli. From a functional standpoint, it manifests as an inability of neurons to produce proper amounts of neuropeptides, like substance P (SP) and calcitonin gene-related peptide (CGRP), in response to tissue injury.

Pre-clinical cell therapy studies showed promising results for treatment of DNP. In a T1DM rat model, MSCs ameliorated hypoalgesia and improved nerve conduction velocity, sciatic nerve blood flow, and capillary number-to-muscle fiber ratio in soleus muscles [50]. MSCs showed the potential to support direct de novo regeneration of neuronal cells [51]. However, paracrine properties seem to be prevalent. Indeed, MSCs produce a list of anti-inflammatory cytokines, which moderate leukocyte recruitment, together with neurotrophic and angiogenic factors such as nerve growth factor (NGF), VEGF, and IGF-1 [52]. This dual action on nerves and vessels is also exerted by EPCs. In a T1DM mouse model, EPC administration increased VEGF, basic fibroblast growth factor (bFGF), and glioma-associated oncogene family zinc finger 1 (Gli 1) protein, which was associated with increased proliferation of Schwann and endothelial cells. Interestingly, engrafted EPCs tended to co-localize near the vasa nervorum [53]. Differently from other BM cell populations, freshly isolated BMderived mononuclear cells (MNCs) produced prevalent improvements on nerves in a DNP rat model [54]. As reported for other complications, in vivo data support the possibility to explore endogenous cell mobilization as a therapeutic alternative to classical cell therapy. In a rodent model of DNP, intraperitoneal administration of G-CSF improved nerve function, preventing axonal atrophy and demyelination, mainly due to peri-neuronal recruitment of stem cells from BM. However, no fusion with recipient cells or proof of BM cell differentiation into neurons was observed, thus suggesting a paracrine action by recruited cells [55].

Currently, no data from clinical trials are available regarding BM cell therapy for treatment of DNP in patients.

\section{Macrovascular Complications}

Critical limb ischemia (CLI), the end stage of peripheral artery disease (PAD), is caused by severe obstruction of blood flow resulting in unbearable pain, ulcers, and a high risk for amputation. Surgical bypass or percutaneous revascularization, the gold standard for the treatment of CLI, exerts only temporary symptomatic alleviation. Furthermore, around one third of patients with CLI cannot be revascularized because of multivascular disease or occlusions of small-caliber blood vessels [56]. Gene and cell therapies have been therefore proposed as a possible complement or alternative to interventional angioplasty.

Different pre-clinical and clinical trials have been carried out in the recent years, mainly using BM-derived MNCs or EPCs, with mixed results. A recent systematic review of clinical trials with intramuscular delivery of BM-derived MNCs in patients with CLI indicates that there is no evidence to support this therapeutic approach. Only in one study was a lower rate of amputation appreciated, pointing out that larger randomized controlled trials are needed in order to provide adequate statistical power [57]. On the other hand, another systematic review identified 13 studies with proper controlled groups using autologous transplantation of BM-derived stem cells. Taken together, these studies suggest BM cells were well tolerated and improved ischemic symptoms in patients with $\mathrm{DM}$, prolonging the amputation-free survival period and promoting complete wound healing [58]. A recent double-blind, randomized, placebo-controlled, phase I/II study showed MSCs ameliorate the ankle brachial pressure index (ABPI) and ankle pressure in patients with established CLI [59]. Conversely, autologous supplementation of CD133+ cells through mobilization showed poor results due to senescence of the stem cell compartment [60].

BM-derived stem cells have been also thoroughly investigated in randomized clinical trials for stroke or acute myocardial infarction (AMI) treatment. Despite differences on the timing of cell transplantation after stroke onset, on the shortor long-term follow-up, and on cell-culture conditions, all studies have led to a significant functional improvement, without adverse effects [61]. However, the limited number of participants enrolled in those studies, associated with limited bioavailability of BM stem cells in persons with $\mathrm{DM}$, demands further investigation to overcome these issues [61]. In AMI treatment, the majority of clinical trials used BM MNCs [62, 63]. Gathered results demonstrated BM MNCs to be safe on the long term, but modest hemodynamic improvements were appreciated [63]. Additionally, MSCs have been used in 
clinical AMI trials. Although their use is safe, no definitive results have been achieved yet [64].

\section{The Downside: Evidence for Impairment of BM-Derived Stem Cells Contributing to Diabetic Complications}

The BM is emerging as just one additional tissue stricken by complications of DM. Initial data from pre-clinical and clinical studies indicated DM causes quantitative and qualitative impairments in circulating stem cells [65]. However, initial damage directly occurs at the source-tissue level, before cells are mobilized to the peripheral circulation, due to a remarkable remodeling of all the components of the marrow niche [66••]. These changes lead to the disruption of stem cell homeostasis, providing a further mechanism to explain and interpret peripheral complications.

\section{Bone Marrow Microangiopathy}

The BM is perfused by a complex network of vessels, formed by penetrating arteries, distal arterioles, and sinusoidal capillaries. Sinusoidal capillaries form highly branched and irregular networks and are primarily found between hematopoietic cells within the marrow cavity. Arteries contain comparably few side branches and are mainly associated with boneforming cells, i.e., osteoblasts. Our seminal work provided the first evidences that diabetic BM undergoes profound structural alterations [67]. Using a T1DM mouse model, we reported a severe microvascular rarefaction responsible for marrow hypoperfusion. Isolated endothelial cells from diabetic mice presented higher levels of ROS production and upregulation of the senescence marker $\beta$-galactosidase [67]. Endothelial dysfunction was confirmed in a subsequent study showing an increased vascular leakage of proteins and leukocytes, both hallmarks of vascular diabetic complications. This was associated with an increased activity of redox-sensitive RhoA GTPase and downstream kinase ROCK 1/2. Both RhoA silencing with a dominant negative form and ROCK inhibition rescued endothelial dysfunction, restoring Akt-dependent production of angiocrine factors, and vascular permeability [68•]. DM also affected the stem cell compartment as HSCs were mostly depleted in the extended, low-perfused part of the marrow [67]. Those findings were confirmed in a T2DM mouse model [69], as well as in people with T2DM [66••]. Histopathological measurements of human BM documented microvascular rarefaction at the sinusoid and arteriole levels, osteopenia, and increased adipogenesis [66••]. Molecular studies highlighted the downregulation of miRNA-155, a master regulator of HSC self-renewal, to be a key underpinning mechanism of diabetic CD34+ cell pauperization [66••]. The occurrence of remodeling of source tissue may represent a serious obstacle to efficient harvesting or mobilization in view of autologous transplantation of CD34+ cells. In fact, a recent study in participants with refractory angina has shown that the efficacy of this treatment is clearly dose dependent [70]. This concept is strengthened by the observation of a delayed repopulating capacity of diabetic BM CD34+ cells [71].

\section{BM Neuropathy and Mobilopathy}

Another master regulator of stem cell homeostasis and trafficking is represented by the BM sensorial and autonomic innervation. HSC release into the bloodstream is regulated by a circadian cycle through the activity of noradrenergic fibers [72]. Sympathetic and nociceptive fibers stimulate stem cell release either directly or indirectly through modulation of stromal cells, i.e., perivascular cells and osteoblasts [73]. Katayama et al. recently showed that HSC mobilization by G-CSF was reduced in sympathectomized mice [74]. They also showed that G-CSF attenuates osteoblast function, via the sympathetic nervous system, thereby facilitating HSC detachment from the endosteal niche. In addition, human CD34+ cells express $\beta 2$-adrenergic and nociceptive receptors that are upregulated by G-CSF [75]. These studies demonstrate that neurotransmitters serve as direct chemoattractants to HSCs under conditions where the mobilization of regenerative cells is required to promote healing of injured or ischemic peripheral tissues [71]. In fact, abrogation of nociceptive signaling by genetic or pharmacological manipulation results in delayed recovery from ischemia [73]. Likewise, the occurrence of autonomic neuropathy accounts for the known defective mobilization of HSCs in persons with DM [76, 77]. A clinical trial (https://www.clinicaltrials.gov, NCT01102699) demonstrated that participants with DM challenged with GCSF have a reduced mobilization of HSCs, due to altered expression of dipeptidyl peptidase-4 (DPP4, also known as adenosine deaminase complexing protein 2 or CD26) [78, 79]. DPP-4's natural substrate is the chemokine SDF-1 $\alpha$, a major regulator of the stem/progenitor cell trafficking between the $\mathrm{BM}$ and circulation. A meta-analysis review on clinical trials pointed out that G-CSF is the main affected mobilization signaling pathway in people with DM [80]. Several studies in animal models replicated the human findings and provided an insight for the underlying mechanisms [81]. In particular, Albiero et al. demonstrated that both experimental murine models of T1DM and T2DM develop BM autonomic neuropathy with an impaired mobilization of HSCs. This effect is mediated by upregulation of p66Shc and downregulation of Sirtuin 1 (Sirt1), together leading to elevated expression of the adhesion molecule CD62L (L-selectin). Knock-out of p66Shc, as well as overexpression of Sirt1, was able to restore proper stem cell trafficking [82•]. In our recent study, we demonstrated a rarefaction in nociceptive fibers in the BM of patients with T2DM [83••]. This was associated with a drastic 
reduction of NK1R, the receptor for nociceptive transmitter SP [83••]. In addition, following induction of limb ischemia, diabetic mice showed an incapacity to create a peripheral-toBM SP gradient. As said before, this gradient drives the mobilization and homing of HSCs to the ischemic site [73]. Consequently, there was a reduction in the recruitment of NK1R+ HSCs to the ischemic limb muscles of diabetic mice, resulting in delayed recovery and depressed reparative angiogenesis [83••]. Collectively, these reports indicate that neuropathy conjointly with microangiopathy may adversely affect stem cell-based therapies in patients with DM.

\section{Adipogenesis and Bone Resorption}

Along with microangiopathy and autonomic neuropathy, increased adipose tissue is a hallmark of BM remodeling in DM, both in animal models and humans [66••, 67]. These changes are likely due to enhanced adipogenesis from MSCs [84]. This is associated both in humans and animal models with a decrease of the ratio between trabecular bone mass and adipose tissue $[66 \bullet \cdot, 67]$. Increased deposition of adipose tissue, as well as a reduction in the number of osteoblasts, could be enlisted among the causes of stem cell pauperization observed in diabetic BM. In fact, adipocytes can disturb the cellular network inside the niches by releasing oxidative and inflammatory mediators [85], activating the AGE pathway [86], and modifying the metabolic milieu [87].

\section{BM-Derived Stem Cells Involved in Other Complications}

In previous sections, we have illustrated how DM complications extend to the BM and impact on stem cells and their niche. The movement of damaged cells along with a spectrum of lineage-committed cells into the circulation may pave the way for the enhancement of peripheral complications. This assumption is supported by new experimental evidence showing that BM-derived cells can actively participate at the two different stages of DR. In the non-proliferative stage, BMderived CD11b-positive cells contribute to capillary degeneration through leukostasis [88]. As demonstrated by Li et al., BM-mobilized monocytes can contribute to the generation of oxidative stress and vessel regression [89]. On the other hand, BM-derived EPCs play a pivotal role in the angiogenic stage of DR via the secretion of growth factors and chemokines [90]. Studies investigating the correlation of EPC numbers and their contribution to DR in humans produced mixed results. For instance, CD34+ cells were found either increased, decreased, or unchanged in T2DM-associated DR [91, 92], whereas in T1DM, EPC numbers correlate well with the proliferative grade of the disease $[93,94]$. With regard to diabetic nephropathy, clinical studies pointed out a correlation between microalbuminuria and circulating CD34+ cells $[95,96]$. Only pre-clinical data are available regarding diabetic neuropathy.
In an elegant study using a T1DM animal model, Terashima et al. showed that BM cells are indeed involved in the pathobiology of diabetic neuropathy, being able to fuse with neurons in the dorsal root ganglia [97]. In a following study, they found similar results in diabetic animals, demonstrating these fusion events occur between neuronal cells and a specific subset of HSCs expressing insulin and TNF- $\alpha$ [98•]. The link between BM pathology and vasculopathy seemingly involves the spleen as a mandatory site of cell commitment to an inflammatory phenotype. It is well known that patients with acute coronary syndromes have a high risk of recurrent events. This was ascribed to mobilization of myeloid cells that have disruptive activity of atherosclerotic plaques. These hematopoietic stem and progenitor cells are liberated from BM niches via sympathetic nervous system signaling. They then colonize the spleen, yielding a sustained boost in monocyte production [99].

\section{Restore BM Functionality to Improve Cell Therapy of Diabetic Complications}

Data reported in previous sections suggest that DM poses serious challenges to current stem cell therapies. The obvious approach to interrupt the vicious cycle between peripheral and $\mathrm{BM}$ damage is to reinforce classical interventions for achievement of proper and consistent metabolic control (Fig. 1). Welldesigned clinical trials similar to the UK Prospective Diabetes Study should be performed to establish the association between stable normalization of hyperglycemia and improvement of BM pathology. Performing such a study will be complicated by the need of an invasive procedure to collect BM material. Alternatively, analyses of circulating cells could be used as a surrogate endpoint. The use of ROS scavengers could also provide opportunities. Studies in experimental models indicate that abrogation of $\mathrm{p} 66 \mathrm{Shc}$ results in protection from angiotensin II-induced cardiac damage [100], improvement of reparative angiogenesis and reduction of myocyte apoptosis following induction of limb ischemia, and preservation of satellite cell proliferation and differentiation under in vitro conditions of high oxidative stress [101, 102]. Blockade of this pathway by specific inhibitors of protein kinase $C \beta$ (PKC $\beta$ ), the enzyme responsible for p66Shc phosphorylation/activation, was demonstrated to ameliorate the vascular dysfunction in diabetic animals, providing a new strategy for the treatment of diabetic vascular complications $[103,104]$. Indeed, the PKC $\beta$ inhibitor LY333531, also known as ruboxistaurin, is under advanced investigation in clinical trials to treat DR. It would be interesting to investigate if $\mathrm{PKC} \beta$ inhibitors are useful in preventing or rescuing BM stem cell availability and functionality in DM.

Thiamine pyrophosphate is the cofactor for transketolase, the rate-limiting enzyme that shunts glyceraldehyde 3- 
Fig. 1 Mechanisms responsible for bone marrow remodeling under chronic hyperglycemia and oxidative stress and potential therapeutic applications. The orange box represents early pathobiological events which trigger later ones, shown in the yellow boxes. Possible therapeutic applications are shown in the green boxes

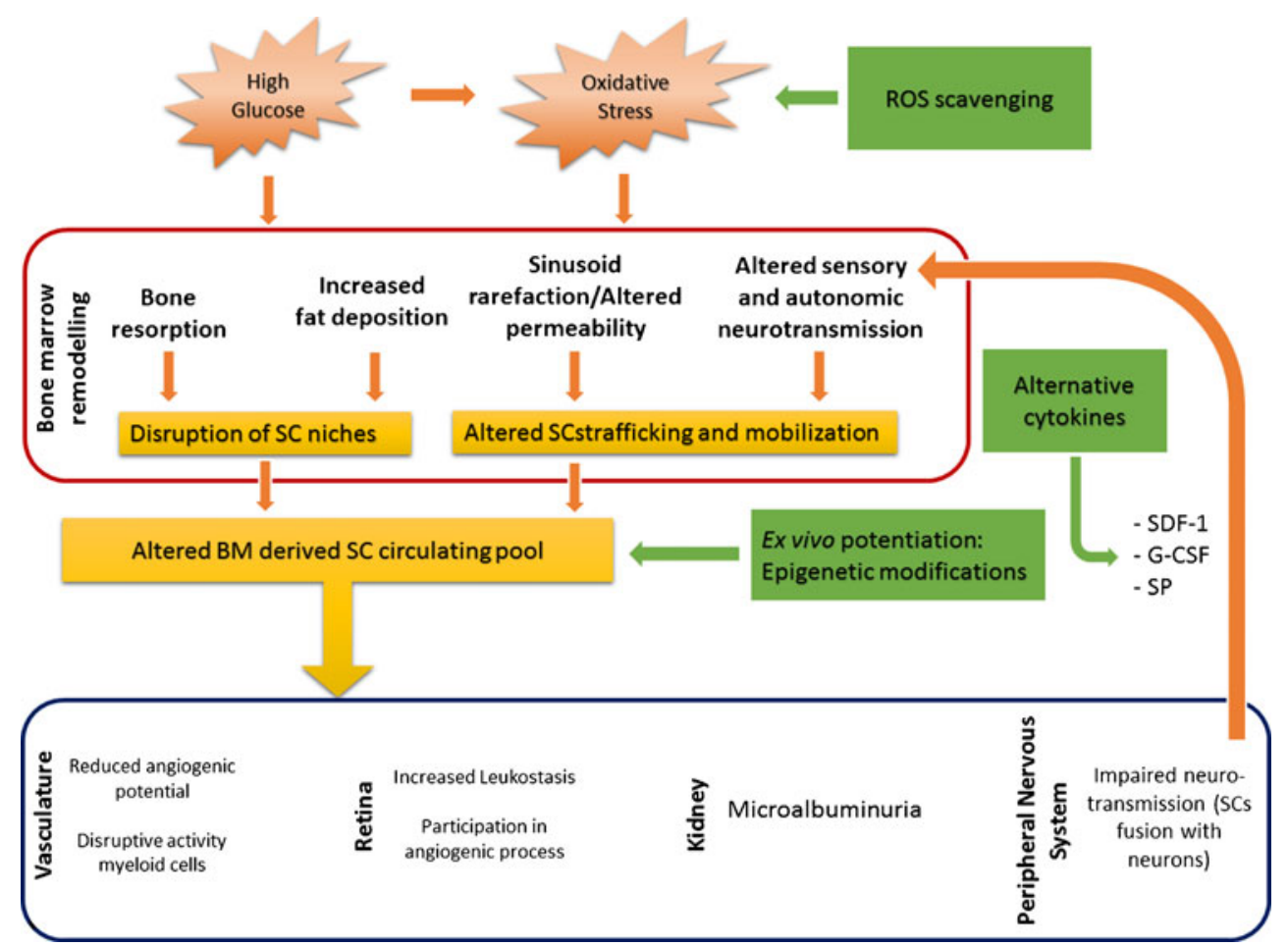

phosphate and fructose 6-phosphate from glycolysis into the non-oxidative branch of the pentose phosphate pathway. Thiamine deficiency has been reported in diabetes, and correction of the defect by supplementation of thiamine or its derivative, benfotiamine, was shown to protect against diabetic nephropathy and retinal microangiopathy [105]. We have also shown that benfotiamine supplementation prevented microangiopathy and hypoperfusion of diabetic BM. Furthermore, benfotiamine reduced oxidative stress and oxidative damage of DNA in BM cells. Importantly, these effects were associated with prevention of HSC depletion, both in terms of absolute number and relative proportion to total BM cells, and inhibition of apoptosis [67]. Clinical studies using benfotiamine are therefore eagerly waited. Moreover, similar strategies could be useful to counteract neuropathy and related insensitivity to chemokine stimulation, e.g., mobilopathy [82•].

Mobilopathy could also benefit by the use of alternative cytokines different from G-CSF. In a retrospective study, Fadini et al. observed that inability of G-CSF to mobilize stem cells in patients with DM was abrogated when administrated in conjunction with pleraxifor, an antagonist of the SDF-1/ CXCR4 axis [106•]. Moreover, our study on nociceptive signaling paves the way for the application of modulators of pain as a novel strategy to help proper mobilization [83••].

Alternatively, specific treatment on ex vivo isolated cells could help to restore their full regenerative potential. As discussed earlier, BM-derived cells can be isolated and expanded ex vivo for autologous or allogeneic transplantation.
Gene therapy could be a viable approach in order to rescue stem cell functionality. For instance, the microRNA-155 downregulation in HSCs represents a candidate target to maintain the pool of original stem cells in diabetic BM [66••]. Conversely, silencing of microRNA-15a and microRNA-16 could ameliorate EPC angiogenic potential [107]. A less problematic approach would be the pre-conditioning of BMderived stem cells in order to improve their regenerative potential [108]. Pre-conditioning with anoxic microenvironment increased the cardio-protective properties of MSCs in a model of diabetic cardiomyopathy [109]. Supplementing the culture media with a cocktail of potentiating cytokines could also be a viable option $[110,111]$. However, it has to be taken into account that transplanted stem cells, either fortified autologous cells or healthy allogeneic cells, have to face a diabetic milieu which can obliterate any salutary improvement.

\section{Conclusions}

In summary, BM cell therapy represents a potential opportunity for treatment of diabetic complications, yet its potential is not fully explored and realized. Even in the case in which this regenerative approach will prove to be marginally useful in a clinical setting, the concept of restoring the proper BM environment remains of vital importance and requires attention for tailoring novel therapies capable of restating global organ homeostasis in patients with DM. 
Acknowledgments This study was supported by the program grant "Unravelling mechanisms of stem cell depletion for preservation of regenerative" founded by British Heart Foundation.

\section{Compliance with Ethical Standards}

Conflict of Interest Giuseppe Mangialardi and Paolo Madeddu declare that they have no conflict of interest.

Human and Animal Rights and Informed Consent This article does not contain any studies with human or animal subjects performed by any of the authors.

Open Access This article is distributed under the terms of the Creative Commons Attribution 4.0 International License (http:// creativecommons.org/licenses/by/4.0/), which permits unrestricted use, distribution, and reproduction in any medium, provided you give appropriate credit to the original author(s) and the source, provide a link to the Creative Commons license, and indicate if changes were made.

\section{References}

Papers of particular interest, published recently, have been highlighted as:

- Of importance

•• Of major importance

1. Kayama Y, Raaz U, Jagger A, et al. Diabetic cardiovascular disease induced by oxidative stress. Int J Mol Sci. 2015;16:25234 63.

2. Bottomley JM, Raymond FD. Pharmaco-economic issues for diabetes therapy. Best practice \& research. Clin Endocrinol Metab. 2007;21:657-85.

3. Assmus B, Dimmeler S, Zeiher AM. Cardiac cell therapy: lost in meta-analyses. Circ Res. 2015;116:1291-2.

4. Quesenberry PJ, Goldberg LR, Dooner MS. Concise reviews: a stem cell apostasy: a tale of four H words. Stem Cells. 2015;33: 15-20.

5. Babovic S, Eaves CJ. Hierarchical organization of fetal and adult hematopoietic stem cells. Exp Cell Res. 2014;329:185-91.

6. Alenzi FQ, Alenazi BQ, Ahmad SY, et al. The haemopoietic stem cell: between apoptosis and self-renewal. Yale J Biol Med. 2009;82:7-18.

7. Takahashi T, Kalka C, Masuda H, et al. Ischemia- and cytokineinduced mobilization of bone marrow-derived endothelial progenitor cells for neovascularization. Nat Med. 1999;5:434-8.

8. Kusumbe AP, Ramasamy SK, Adams RH. Coupling of angiogenesis and osteogenesis by a specific vessel subtype in bone. Nature. 2014;507:323-8.

9. Dominici M, Le Blanc K, Mueller I, et al. Minimal criteria for defining multipotent mesenchymal stromal cells. The International Society for Cellular Therapy position statement. Cytotherapy. 2006;8:315-7.

10. Bara JJ, Richards RG, Alini M, et al. Concise review: bone marrow-derived mesenchymal stem cells change phenotype following in vitro culture: implications for basic research and the clinic. Stem Cells. 2014;32:1713-23.

11. Chen L, Tredget EE, Wu PY, et al. Paracrine factors of mesenchymal stem cells recruit macrophages and endothelial lineage cells and enhance wound healing. PLoS One. 2008;3:e1886.
12. Ishikawa M, Asahara T. Endothelial progenitor cell culture for vascular regeneration. Stem Cells Dev. 2004;13:344-9.

13. Desai A, Glaser A, Liu D, et al. Microarray-based characterization of a colony assay used to investigate endothelial progenitor cells and relevance to endothelial function in humans. Arterioscler Thromb Vasc Biol. 2009;29:121-7.

14. Yoder MC. Is endothelium the origin of endothelial progenitor cells? Arterioscler Thromb Vasc Biol. 2010;30:1094-103.

15. Zhang M, Malik AB, Rehman J. Endothelial progenitor cells and vascular repair. Curr Opin Hematol. 2014;21:224-8.

16. Wan Y, Lu C, Cao J, et al. Osteoblastic Wnts differentially regulate bone remodeling and the maintenance of bone marrow mesenchymal stem cells. Bone. 2013;55:258-67.

17. Pettit AR, Chang MK, Hume DA, et al. Osteal macrophages: a new twist on coupling during bone dynamics. Bone. 2008;43: 976-82.

18. Kobayashi H, Butler JM, O'Donnell R, et al. Angiocrine factors from Akt-activated endothelial cells balance self-renewal and differentiation of haematopoietic stem cells. Nat Cell Biol. 2010;12: 1046-56.

19. Corselli M, Chin CJ, Parekh C, et al. Perivascular support of human hematopoietic stem/progenitor cells. Blood. 2013;121: 2891-901.

20. Mangialardi G, Spinetti G, Reni C, et al. Reactive oxygen species adversely impacts bone marrow microenvironment in diabetes. Antioxid Redox Signal. 2014;21:1620-33. An extensive review on reactive oxygen species role in bone marrow niche homeostasis and its disruption in diabetes.

21. Klein BE. Overview of epidemiologic studies of diabetic retinopathy. Ophthalmic Epidemiol. 2007;14:179-83.

22. Semeraro F, Cancarini A, dell'Omo R, et al. Diabetic retinopathy: vascular and inflammatory disease. J Diabetes Res. 2015;2015: 582060 .

23. Caplan AI. Adult mesenchymal stem cells: when, where, and how. Stem Cells Int. 2015;2015:628767.

24. Wang S, Lu B, Girman S, et al. Non-invasive stem cell therapy in a rat model for retinal degeneration and vascular pathology. PLoS One. 2010;5:e9200.

25. Abcouwer SF, Gardner TW. Diabetic retinopathy: loss of neuroretinal adaptation to the diabetic metabolic environment Ann N Y Acad Sci. 2014;1311:174-90.

26. Siqueira RC, Messias A, Messias K, et al. Quality of life in patients with retinitis pigmentosa submitted to intravitreal use of bone marrow-derived stem cells (Reticell -clinical trial). Stem Cell Res Ther. 2015;6:29.

27. Caballero S, Sengupta N, Afzal A, et al. Ischemic vascular damage can be repaired by healthy, but not diabetic, endothelial progenitor cells. Diabetes. 2007;56:960-7.

28. Park SS, Caballero S, Bauer G, et al. Long-term effects of intravitreal injection of GMP-grade bone-marrow-derived CD34+ cells in NOD-SCID mice with acute ischemia-reperfusion injury. Invest Ophthalmol Vis Sci. 2012;53:986-94.

29. Park SS, Bauer G, Abedi M, et al. Intravitreal autologous bone marrow $\mathrm{CD} 34+$ cell therapy for ischemic and degenerative retinal disorders: preliminary phase 1 clinical trial findings. Invest Ophthalmol Vis Sci. 2015;56:81-9.

30. Siqueira RC, Messias A, Gurgel VP, et al. Improvement of ischaemic macular oedema after intravitreal injection of autologous bone marrow-derived haematopoietic stem cells. Acta Ophthalmol (Copenh). 2015;93:e174-6.

31. Seferovic PM, Paulus WJ. Clinical diabetic cardiomyopathy: a two-faced disease with restrictive and dilated phenotypes. Eur Heart J. 2015;36:1718-27. 1727a-1727c.

32. Bugger H, Abel ED. Molecular mechanisms of diabetic cardiomyopathy. Diabetologia. 2014;57:660-71. 
33. Nagaya N, Kangawa K, Itoh T, et al. Transplantation of mesenchymal stem cells improves cardiac function in a rat model of dilated cardiomyopathy. Circulation. 2005;112:1128-35.

34. Mias C, Lairez O, Trouche E, et al. Mesenchymal stem cells promote matrix metalloproteinase secretion by cardiac fibroblasts and reduce cardiac ventricular fibrosis after myocardial infarction. Stem Cells. 2009;27:2734-43.

35. Dong X, Zhu F, Liu Q, et al. Transplanted bone marrow mesenchymal stem cells protects myocardium by regulating 14-3-3 protein in a rat model of diabetic cardiomyopathy. Int J Clin Exp Pathol. 2014;7:3714-23.

36. Ammar HI, Sequiera GL, Nashed MB, et al. Comparison of adipose tissue- and bone marrow-derived mesenchymal stem cells for alleviating doxorubicin-induced cardiac dysfunction in diabetic rats. Stem Cell Res Ther. 2015;6:148.

37. Lim YH, Joe JH, Jang KS, et al. Effects of granulocyte-colony stimulating factor (G-CSF) on diabetic cardiomyopathy in Otsuka Long-Evans Tokushima fatty rats. Cardiovasc Diabetol. 2011;10: 92.

38. Ascione R, Rowlinson J, Avolio E, et al. Migration towards SDF-1 selects angiogenin-expressing bone marrow monocytes endowed with cardiac reparative activity in patients with previous myocardial infarction. Stem Cell Res Ther. 2015;6:53.

39. Kishore R, Verma SK, Mackie AR, et al. Bone marrow progenitor cell therapy-mediated paracrine regulation of cardiac miRNA-155 modulates fibrotic response in diabetic hearts. PLoS One. 2013;8: e60161.

40. Zhu K, Li J, Wang Y, et al. Intramyocardial autologous bone marrow-derived stem cells injection for ischemic heart disease ineligible for revascularization: a systematic review and metaanalysis. Arch Med Res. 2015;46:286-95.

41. Lee RH, Seo MJ, Reger RL, et al. Multipotent stromal cells from human marrow home to and promote repair of pancreatic islets and renal glomeruli in diabetic NOD/scid mice. Proc Natl Acad Sci U S A. 2006;103:17438-43.

42. Ezquer F, Ezquer M, Simon V, et al. Endovenous administration of bone-marrow-derived multipotent mesenchymal stromal cells prevents renal failure in diabetic mice. Biol Blood Marrow Transplant J Am Soc Blood Marrow Transplant. 2009;15:1354-65.

43. Wang S, Li Y, Zhao J, et al. Mesenchymal stem cells ameliorate podocyte injury and proteinuria in a type 1 diabetic nephropathy rat model. Biol Blood Marrow Transplant J Am Soc Blood Marrow Transplant. 2013;19:538-46.

44. Lv S, Cheng J, Sun A, et al. Mesenchymal stem cells transplantation ameliorates glomerular injury in streptozotocin-induced diabetic nephropathy in rats via inhibiting oxidative stress. Diabetes Res Clin Pract. 2014;104:143-54.

45. Lv SS, Liu G, Wang JP, et al. Mesenchymal stem cells transplantation ameliorates glomerular injury in streptozotocin-induced diabetic nephropathy in rats via inhibiting macrophage infiltration. Int Immunopharmacol. 2013;17:275-82.

46. Abdel Aziz MT, Wassef MA, Ahmed HH, et al. The role of bone marrow derived-mesenchymal stem cells in attenuation of kidney function in rats with diabetic nephropathy. Diabetol Metab Syndr. 2014;6:34.

47. Lv S, Liu G, Sun A, et al. Mesenchymal stem cells ameliorate diabetic glomerular fibrosis in vivo and in vitro by inhibiting TGF-beta signalling via secretion of bone morphogenetic protein 7. Diab Vasc Dis Res. 2014;11:251-61.

48. Zhang Y, Yuen DA, Advani A, et al. Early-outgrowth bone marrow cells attenuate renal injury and dysfunction via an antioxidant effect in a mouse model of type 2 diabetes. Diabetes. 2012;61: 2114-25.

49. Song SM, Wang CC, Qi SH, et al. Angiotensin receptor blockade attenuates glomerulosclerosis progression by promoting VEGF expression and bone marrow-derived cells recruitment. Nephrol
Dial Transplant Off Publ Eur Dial Transplant Assoc_-Eur Renal Assoc. 2012;27:2712-9.

50. Shibata T, Naruse K, Kamiya H, et al. Transplantation of bone marrow-derived mesenchymal stem cells improves diabetic polyneuropathy in rats. Diabetes. 2008;57:3099-107.

51. Lin R, Ding Z, Ma H, et al. In vitro conditioned bone marrowderived mesenchymal stem cells promote de novo functional enteric nerve regeneration, but not through direct-transdifferentiation. Stem Cells. 2015;33(12):3545-57.

52. Hsieh JY, Wang HW, Chang SJ, et al. Mesenchymal stem cells from human umbilical cord express preferentially secreted factors related to neuroprotection, neurogenesis, and angiogenesis. PLoS One. 2013;8:e72604.

53. Jeong JO, Kim MO, Kim H, et al. Dual angiogenic and neurotrophic effects of bone marrow-derived endothelial progenitor cells on diabetic neuropathy. Circulation. 2009;119:699-708.

54. Naruse K, Sato J, Funakubo M, et al. Transplantation of bone marrow-derived mononuclear cells improves mechanical hyperalgesia, cold allodynia and nerve function in diabetic neuropathy. PLoS One. 2011;6:e27458.

55. Kim KS, Song YS, Jin J, et al. Granulocyte-colony stimulating factor as a treatment for diabetic neuropathy in rat. Mol Cell Endocrinol. 2015;414:64-72.

56. Criqui MH, Aboyans V. Epidemiology of peripheral artery disease. Circ Res. 2015;116:1509-26.

57. Moazzami K, Moazzami B, Roohi A, et al. Local intramuscular transplantation of autologous mononuclear cells for critical lower limb ischaemia. Cochrane Database Syst Rev. 2014;12: CD008347.

58. Powell RJ, Marston WA, Berceli SA, et al. Cellular therapy with Ixmyelocel-T to treat critical limb ischemia: the randomized, double-blind, placebo-controlled RESTORE-CLI trial. Mol Ther J Am Soc Gene Ther. 2012;20:1280-6.

59. Gupta PK, Chullikana A, Parakh R, et al. A double blind randomized placebo controlled phase I/II study assessing the safety and efficacy of allogeneic bone marrow derived mesenchymal stem cell in critical limb ischemia. J Transl Med. 2013;11:143.

60. Raval AN, Schmuck EG, Tefera G, et al. Bilateral administration of autologous CD133+ cells in ambulatory patients with refractory critical limb ischemia: lessons learned from a pilot randomized, double-blind, placebo-controlled trial. Cytotherapy. 2014;16: 1720-32.

61. Jeong H, Yim HW, Cho YS, et al. Efficacy and safety of stem cell therapies for patients with stroke: a systematic review and single arm meta-analysis. Int J Stem Cells. 2014;7:63-9.

62. Assmus B, Leistner DM, Schachinger V, et al. Long-term clinical outcome after intracoronary application of bone marrow-derived mononuclear cells for acute myocardial infarction: migratory capacity of administered cells determines event-free survival. Eur Heart J. 2014;35:1275-83.

63. Prasad K, Sharma A, Garg A, et al. Intravenous autologous bone marrow mononuclear stem cell therapy for ischemic stroke: a multicentric, randomized trial. Stroke. 2014;45:3618-24.

64. Lalu MM, McIntyre L, Pugliese C, et al. Safety of cell therapy with mesenchymal stromal cells (SafeCell): a systematic review and meta-analysis of clinical trials. PLoS One. 2012;7:e47559.

65. Menegazzo L, Albiero M, Avogaro A, et al. Endothelial progenitor cells in diabetes mellitus. Biofactors. 2012;38:194-202.

66.• Spinetti G, Cordella D, Fortunato O, et al. Global remodeling of the vascular stem cell niche in bone marrow of diabetic patients: implication of the microRNA-155/FOXO3a signaling pathway. Circ Res. 2013;112:510-22. An extensive study on bone marrow remodelling in type 2 diabetic patients.

67. Oikawa A, Siragusa M, Quaini F, et al. Diabetes mellitus induces bone marrow microangiopathy. Arterioscler Thromb Vasc Biol. 2010;30:498-508. 
68. Mangialardi G, Katare R, Oikawa A, et al. Diabetes causes bone marrow endothelial barrier dysfunction by activation of the RhoARho-associated kinase signaling pathway. Arterioscler Thromb Vasc Biol. 2013;33:555-64. This study demonstrates that diabetic oxidative stress alters stem cell trafficking properties in bone marrow vasculature.

69. Orlandi A, Chavakis E, Seeger F, et al. Long-term diabetes impairs repopulation of hematopoietic progenitor cells and dysregulates the cytokine expression in the bone marrow microenvironment in mice. Basic Res Cardiol. 2010;105:703-12.

70. Povsic TJ, Junge C, Nada A, et al. A phase 3, randomized, doubleblinded, active-controlled, unblinded standard of care study assessing the efficacy and safety of intramyocardial autologous CD34+ cell administration in patients with refractory angina: design of the RENEW study. Am Heart J. 2013;165:854-61. e852.

71. Ferraro F, Lymperi S, Mendez-Ferrer S, et al. Diabetes impairs hematopoietic stem cell mobilization by altering niche function. Sci Transl Med. 2011;3:104ra101.

72. Mendez-Ferrer S, Lucas D, Battista M, et al. Haematopoietic stem cell release is regulated by circadian oscillations. Nature. 2008;452:442-7.

73. Amadesi S, Reni C, Katare R, et al. Role for substance p-based nociceptive signaling in progenitor cell activation and angiogenesis during ischemia in mice and in human subjects. Circulation. 2012;125(125):1774-86. S1771-1719.

74. Katayama Y, Battista M, Kao WM, et al. Signals from the sympathetic nervous system regulate hematopoietic stem cell egress from bone marrow. Cell. 2006;124:407-21.

75. Spiegel A, Shivtiel S, Kalinkovich A, et al. Catecholaminergic neurotransmitters regulate migration and repopulation of immature human CD34+ cells through Wnt signaling. Nat Immunol. 2007;8:1123-31.

76. Turan RG, Turan CH, Bozdag-Turan I, et al. Impaired mobilization of $\mathrm{CD} 133(+)$ bone marrow-derived circulating progenitor cells with an increased number of diseased coronary arteries in ischemic heart disease patients with diabetes. Circ J Off J Jpn Circ Soc. 2011;75:2635-41.

77. Ling L, Shen Y, Wang K, et al. Worse clinical outcomes in acute myocardial infarction patients with type 2 diabetes mellitus: relevance to impaired endothelial progenitor cells mobilization. PLoS One. 2012;7:e50739.

78. Fadini GP, Avogaro A. Dipeptidyl peptidase-4 inhibition and vascular repair by mobilization of endogenous stem cells in diabetes and beyond. Atherosclerosis. 2013;229:23-9.

79. Fadini GP, Albiero M, Seeger F, et al. Stem cell compartmentalization in diabetes and high cardiovascular risk reveals the role of DPP-4 in diabetic stem cell mobilopathy. Basic Res Cardiol. 2013;108:313.

80. Fadini GP, Avogaro A. Diabetes impairs mobilization of stem cells for the treatment of cardiovascular disease: a meta-regression analysis. Int J Cardiol. 2013;168:892-7.

81. Westerweel PE, Teraa M, Rafii S, et al. Impaired endothelial progenitor cell mobilization and dysfunctional bone marrow stroma in diabetes mellitus. PLoS One. 2013;8:e60357.

82. Albiero M, Poncina N, Tjwa M, et al. Diabetes causes bone marrow autonomic neuropathy and impairs stem cell mobilization via dysregulated p66Shc and Sirt1. Diabetes. 2014;63:1353-65. This study provide a mechanistic insight of diabetic mobilopathy.

83.•• Dang Z, Maselli D, Spinetti G, et al. Sensory neuropathy hampers nociception-mediated bone marrow stem cell release in mice and patients with diabetes. Diabetologia. 2015;58:2653-62. This study provides for the first time findings for nociception fiber involvement in diabetic mobilopathy.

84. Botolin S, McCabe LR. Bone loss and increased bone adiposity in spontaneous and pharmacologically induced diabetic mice. Endocrinology. 2007;148:198-205.
85. Adler BJ, Kaushansky K, Rubin CT. Obesity-driven disruption of haematopoiesis and the bone marrow niche. Nature reviews. Endocrinology. 2014;10:737-48.

86. Dong XN, Qin A, Xu J, et al. In situ accumulation of advanced glycation endproducts (AGEs) in bone matrix and its correlation with osteoclastic bone resorption. Bone. 2011;49:174-83.

87. Ying X, Chen X, Liu H, et al. Silibinin alleviates high glucosesuppressed osteogenic differentiation of human bone marrow stromal cells via antioxidant effect and PI3K/Akt signaling. Eur J Pharmacol. 2015;765:394-401.

88. Serra AM, Waddell J, Manivannan A, et al. CD11b+ bone marrow-derived monocytes are the major leukocyte subset responsible for retinal capillary leukostasis in experimental diabetes in mouse and express high levels of CCR5 in the circulation. Am J Pathol. 2012;181:719-27.

89. Li G, Veenstra AA, Talahalli RR, et al. Marrow-derived cells regulate the development of early diabetic retinopathy and tactile allodynia in mice. Diabetes. 2012;61:3294-303.

90. Liu X, Li Y, Liu Y, et al. Endothelial progenitor cells (EPCs) mobilized and activated by neurotrophic factors may contribute to pathologic neovascularization in diabetic retinopathy. Am J Pathol. 2010;176:504-15.

91. Lee IG, Chae SL, Kim JC. Involvement of circulating endothelial progenitor cells and vasculogenic factors in the pathogenesis of diabetic retinopathy. Eye. 2006;20:546-52.

92. Lombardo MF, Iacopino P, Cuzzola M, et al. Type 2 diabetes mellitus impairs the maturation of endothelial progenitor cells and increases the number of circulating endothelial cells in peripheral blood. Cytometry A J Int Soc Anal Cytol. 2012;81:856-64.

93. Tan K, Lessieur E, Cutler A, et al. Impaired function of circulating CD34(+) CD45(-) cells in patients with proliferative diabetic retinopathy. Exp Eye Res. 2010;91:229-37.

94. Brunner S, Schernthaner GH, Satler M, et al. Correlation of different circulating endothelial progenitor cells to stages of diabetic retinopathy: first in vivo data. Invest Ophthalmol Vis Sci. 2009;50: 392-8.

95. Dessapt C, Karalliedde J, Hernandez-Fuentes M, et al. Circulating vascular progenitor cells in patients with type 1 diabetes and microalbuminuria. Diabetes Care. 2010;33:875-7.

96. Reinhard H, Jacobsen PK, Lajer M, et al. Endothelial progenitor cells in long-standing asymptomatic type 1 diabetic patients with or without diabetic nephropathy. Nephron Clin Pract. 2011;118: c309-14.

97. Terashima T, Kojima H, Chan L. Bone marrow expression of poly(ADP-ribose) polymerase underlies diabetic neuropathy via hematopoietic-neuronal cell fusion. FASEB J Off Publ Fed Am Soc Exp Biol. 2012;26:295-308.

98. Katagi M, Terashima T, Okano J, et al. Hyperglycemia induces abnormal gene expression in hematopoietic stem cells and their progeny in diabetic neuropathy. FEBS Lett. 2014;588:1080-6. One of the most recent publications contributing to explain bone marrow-derived stem cell contribution to diabetic neuropathy.

99. Dutta P, Courties G, Wei Y, et al. Myocardial infarction accelerates atherosclerosis. Nature. 2012;487:325-9.

100. Graiani G, Lagrasta C, Migliaccio E, et al. Genetic deletion of the p66Shc adaptor protein protects from angiotensin II-induced myocardial damage. Hypertension. 2005;46:433-40.

101. Zaccagnini G, Martelli F, Magenta A, et al. p66(ShcA) and oxidative stress modulate myogenic differentiation and skeletal muscle regeneration after hind limb ischemia. J Biol Chem. 2007;282: 31453-9.

102. Natalicchio A, Tortosa F, Labarbuta R, et al. The p66(Shc) redox adaptor protein is induced by saturated fatty acids and mediates lipotoxicity-induced apoptosis in pancreatic beta cells. Diabetologia. 2015;58:1260-71. 
103. Wei L, Yin Z, Yuan Y, et al. A PKC-beta inhibitor treatment reverses cardiac microvascular barrier dysfunction in diabetic rats. Microvasc Res. 2010;80:158-65.

104. Budhiraja S, Singh J. Protein kinase C beta inhibitors: a new therapeutic target for diabetic nephropathy and vascular complications. Fundam Clin Pharmacol. 2008;22:231-40.

105. Hammes HP, Du X, Edelstein D, et al. Benfotiamine blocks three major pathways of hyperglycemic damage and prevents experimental diabetic retinopathy. Nat Med. 2003;9:294-9.

106. Fadini GP, Fiala M, Cappellari R, et al. Diabetes limits stem cell mobilization following G-CSF but not plerixafor. Diabetes. 2015;64:2969-77. A potential mechanism to be exploited in diabetic mobilopathy therapy.

107. Spinetti G, Fortunato O, Caporali A, et al. MicroRNA-15a and microRNA-16 impair human circulating proangiogenic cell functions and are increased in the proangiogenic cells and serum of patients with critical limb ischemia. Circ Res. 2013;112:335-46.

108. Choudhery MS, Khan M, Mahmood R, et al. Mesenchymal stem cells conditioned with glucose depletion augments their ability to repair-infarcted myocardium. J Cell Mol Med. 2012;16:2518-29.

109. Li JH, Zhang N, Wang JA. Improved anti-apoptotic and anti-remodeling potency of bone marrow mesenchymal stem cells by anoxic pre-conditioning in diabetic cardiomyopathy. J Endocrinol Investig. 2008;31:103-10

110. Smith CL, Chaichana KL, Lee YM, et al. Pre-exposure of human adipose mesenchymal stem cells to soluble factors enhances their homing to brain cancer. Stem Cells Transl Med. 2015;4:239-51.

111. Zimmermann JA, McDevitt TC. Pre-conditioning mesenchymal stromal cell spheroids for immunomodulatory paracrine factor secretion. Cytotherapy. 2014;16:331-45. 